\title{
UNIQUENESS IN A MELTING SLAB WITH SPACE- AND TIME-DEPENDENT HEATING*
}

BY

BRUNO A. BOLEY

Cornell University

Summary. Conditions for uniqueness of solution are derived for a slab which melts under an applied heat input dependent both on time and on the melted depth, the melted material being instantaneously removed. Some examples are constructed in which more than one solution is exhibited as well as one in which no melting solution exists. A theorem demonstrating monotonic dependence of solution on boundary data under certain conditions is proved.

Introduction. One of the important problems in the theory of heat conduction with changes of phase is that of a body, initially solid, which melts under a prescribed heat input, the liquid portion being instantaneously removed upon formation. This condition, although not actually realizable in any real physical situation, gives results which are, for short times, identical with those valid when the liquid remains stationary [1] or is ablated at an arbitrary rate [2]; they represent, in the latter case, a lower bound for all times [3], and a good approximation when the Prandtl number of the liquid does not differ too much from unity [4]-[6]. Solutions to this problem for a halfspace were obtained by several authors (for references see for example [7]), while uniqueness proofs were presented in [8] for a halfspace and in [9] for an arbitrary geometrical configuration.

In all the previous solutions heating was applied by means of a prescribed heat input $Q(t)$ applied at the surface of the solid. A physically more meaningful situation arises when the heat is applied either by a time-dependent source radiating energy from a point at some distance from the melting body, or through a heat-conducting layer of liquid; in such cases the heat input depends on position as well as on time. As a consequence, the available uniqueness proofs do not hold, and in fact the solution is no longer always unique, although solutions can still be constructed by the methods in [1] and [10]. It is the primary purpose of the present work to investigate the uniqueness of solution under the circumstances just described, for the one-dimensional case, under an arbitrary heat input $Q(x, t)$.

2. Mathematical formulation. The temperature $T(x, t)$ in the slab $s(t)<x<L$, where the melted thickness is $s(t) \geq 0$, with

$$
s(t)=0 \text { for } t \leq t_{m},
$$

satisfies the heat conduction equation

$$
\frac{\partial}{\partial x}\left[k(T) \frac{\partial T}{\partial x}\right]=\rho c(T) \frac{\partial T}{\partial t}, \quad x>s(t) \geq 0, \quad t>0
$$

under the initial condition

$$
T(x, 0)=T_{0}(x) \leq T_{m}, \quad x>0
$$

* Received November 20, 1968; revised version received December 16, 1968. This work was supported by the Office of Naval Research. 
and the boundary conditions

$$
\left.\begin{array}{rlrl}
T(x, t) & =T_{m} & t & \geq t_{m} \\
-k(T) \frac{\partial T}{\partial x} & =Q(x, t)-\rho l \frac{d s}{d t} & & t>0
\end{array}\right\} \quad x=s(t) .
$$

The symbols $t_{m}, T_{m}, k, \rho, c$ and $l$ stand respectively for the time of start of melting, the melting temperature, the thermal conductivity, the density, the specific heat and the latent heat of fusion. The cold face $x=L$ will here be taken to be insulated, or

$$
\partial T(L, t) / \partial x=0 \quad t>0 .
$$

An overall heat balance may be written from the above equations in the form [8]:

$$
\int_{0}^{t} Q[s(\tau), \tau] d \tau=\int_{s(t)}^{L} H(T) d x+\left(\rho l+H_{m}\right) s(t)
$$

where the heat content $H(T)$, a monotonically increasing function of $T$, is

$$
H(T)=\rho \int_{0}^{T} c\left(T^{\prime}\right) d T^{\prime}, \quad H_{m}=H\left(T_{m}\right) .
$$

3. Uniqueness theorem. It is well known thet the solution is unique before melting starts, and that the same is true after melting, if it is assumed that the melting thickness $s(t)$ is unique [8]. There remains therefore to investigate the conditions under which the latter assumption holds, and this will now be done. The term "solution" will here be taken to indicate a twice continuously differentiable function $T(x, t)$ and a Lipschitz continuous function $s(t)$, of bounded variation. Existence of such a solution is here assumed.

Assume that two solutions (denoted by subscripts 1 and 2 respectively) exist, such that, without loss of generality,

$$
\begin{array}{ll}
s_{1} \equiv s_{2} & 0 \leq t \leq t^{\prime} \quad t^{\prime} \geq t_{m_{1}}, \quad t^{\prime} \geq t_{m_{2}} \\
s_{1}<s_{2} & t^{\prime}<t \leq t^{\prime}+\delta, \quad \delta>0 .
\end{array}
$$

Write Eq. (6) for each of these solutions and subtract the results to obtain (with $t^{\prime}<$ $t \leq t^{\prime}+\delta$ in all that follows):

$$
\begin{aligned}
\int_{t^{\prime}}^{t}\left\{Q\left[s_{2}(\tau), \tau\right]\right. & \left.-Q\left[s_{1}(\tau), \tau\right]\right\} d \tau \\
= & -\int_{s_{1}(t)}^{s_{2}(t)} H_{1} d x+\int_{s_{2}(t)}^{L}\left(H_{2}-H_{1}\right) d x+\left(\rho l+H_{m}\right)\left[s_{2}(t)-s_{1}(t)\right] .
\end{aligned}
$$

From the first of (4), $T_{2}=T_{m}$ and $T_{1} \leq T_{m}$ on $x=s_{2}(t)$, so that the minimum theorem for parabolic equations [8], [11] gives $T_{1} \leq T_{2}$ for $s_{2}(t) \leq x \leq L$, and thus $H_{1} \leq H_{2}$ in this region. Furthermore

$$
\int_{s_{1}}^{s_{2}} H_{1} d x \leq H_{m}\left[s_{2}(t)-s_{1}(t)\right]
$$

so that the right-hand side of (9) is positive. For later use note that in fact

$$
\text { R.H.S. } \geq \rho l\left(s_{2}-s_{1}\right)>0 .
$$


Consider now conditions under which the left-hand side of (9) cannot be positive; under these conditions therefore Eq. (9) is self-contradictory, the assumed conditions (7) cannot be met and the solution is unique. Sufficient conditions for this to be true are:

(a) $Q$ is independent of $x$, or $Q=Q(t)$, since then the left-hand side is zero.

(b) $Q(x, t)$ is a monotonically decreasing function of $x$, since then the left-hand side is negative. This case would arise, for example, if heating were supplied by a source removed from the melting surface, whose effect would decrease with distance from it.

(c) If $Q(x, t)$ is Lipschitz continuous in $x$, that is

$$
\left|Q\left(x_{2}, t\right)-Q\left(x_{1}, t\right)\right| \leq f(t)\left|x_{2}-x_{1}\right|
$$

provided that $f(t)$ is a nonnegative integrable function, then the solution is again unique. Indeed in this case

$$
\begin{aligned}
\int_{t^{\prime}}^{t^{\prime \prime}}\left\{Q\left[s_{2}(\tau), \tau\right]-Q\left[s_{1}(\tau), \tau\right]\right\} d \tau & \leq \int_{t^{\prime}}^{t^{\prime \prime}} f(\tau)\left|s_{2}(\tau)-s_{1}(\tau)\right| d \tau \\
& \leq\left|s_{2}\left(t^{\prime \prime}\right)-s_{1}\left(t^{\prime \prime}\right)\right| f(\tau)\left(t^{\prime \prime}-t^{\prime}\right)
\end{aligned}
$$

provided that $t^{\prime \prime}$ is chosen sufficiently close to $t^{\prime}$, and where the mean-value theorem has been used with $t^{\prime} \leq t \leq t^{\prime \prime}$. There certainly exists a time $t^{\prime \prime}$ sufficiently close to $t^{\prime}$ that the last term in inequality (11) is smaller than the term $\rho l\left[s_{2}\left(t^{\prime \prime}\right)-s_{1}\left(t^{\prime \prime}\right)\right]$, appearing on the right-hand side of Eq. (8). Hence Eq. (9) is again contradicted, and uniqueness is assured. Note that here $Q$ may be an increasing function of $x$.

4. A comparison theorem. It will now be shown that, if two heat inputs $Q_{1}(x, t)$ and $Q_{2}(x, t)$ are applied to the same body, the solution corresponding to each being denoted by the subscript 1 and 2 respectively, then

$$
\begin{array}{cc}
T_{2}(x, t) \geq T_{1}(x, t) ; & s_{2}(t) \leq x \leq L \\
s_{2}(t) \geq s_{1}(t) ; & t \geq 0
\end{array}
$$

provided that

$$
Q_{2}(x, t) \geq Q_{1}(x, t)
$$

and that either one of conditions (a) or (b) at the end of the preceding section hold for both $Q_{1}$ and $Q_{2}$.

This comparison theorem is a direct extension of Theorem IV of Ref. [8], where the $x$-dependence of $Q$ was omitted, and in fact the present proof is quite similar to the one given there.

The proof is given in two parts: it is first shown (I) that the theorem holds immediately, when $s_{2}$ and $s_{1}$ first differ, and then (II) that it cannot fail thereafter. To show part (I), assume, without loss of generality, that a time $t^{\prime}$ exists such that

$$
\begin{array}{ll}
Q_{2}(x, t) \equiv Q_{1}(x, t) & \text { for } \quad 0 \leq t \leq t^{\prime} \\
Q_{2}(x, t)>Q_{1}(x, t) & \text { for } \quad t^{\prime}<t \leq t^{\prime}+\delta, \quad \delta>0 .
\end{array}
$$

Usually, of course, $t^{\prime}=t_{m}$. Assume now that a number $\delta^{\prime}>0$ exists, for which

$$
s_{2}(t)<s_{1}(t) \text { for } t^{\prime}<t \leq t^{\prime}+\delta^{\prime} \leq t^{\prime}+\delta .
$$


Write the heat balance equation (6) for each solution, and subtract to obtain

$$
\begin{aligned}
& \int_{t^{\prime}}^{t}\left\{Q_{2}\left[s_{2}(\tau), \tau\right]-Q_{1}\left[s_{1}(\tau), \tau\right]\right\} d \tau \\
& \quad=\int_{s_{1}(t)}^{L}\left[H\left(T_{2}\right)-H\left(T_{1}\right)\right] d x+\int_{s_{s}(t)}^{s_{1}(t)} H\left(T_{2}\right) d x-\left(\rho l+H_{m}\right)\left[s_{1}(t)-s_{2}(t)\right] .
\end{aligned}
$$

Since, on $x=s_{1}(t), T_{2} \leq T_{m}=T_{1}$, it follows from the minimum theorem [11] that the first integral on the right-hand side is nonpositive. The second integral gives

$$
\int_{s_{2}(t)}^{s_{1}(t)} H\left(T_{2}\right) d x \leq H_{m}\left[s_{1}(t)-s_{2}(t)\right]
$$

so that clearly the entire right-hand side of (15a) is nonpositive. The left-hand side of that equation, however, is positive, in view of (14) and (15), when either condition (a) or (b) of the previous uniqueness theorem holds; hence (15) cannot be true, and part (I) of the proof is complete. Note that the proof would have been valid also if condition (c) of the uniqueness theorem had been enforced; this, however, is not true of the proof for part (II) which follows.

If Eq. (12b) is to fail at some subsequent time there must be at least one time at which $s_{2}=s_{1}$; let the first of these be $t^{\prime \prime}$. Then

$$
T_{2} \geq T_{1} \quad 0<t \leq t^{\prime \prime}
$$

The second of interface conditions (4) gives

$$
-k\left(T_{m}\right) \frac{\partial\left(T_{2}-T_{1}\right)}{\partial x}=\left\{Q_{2}\left[s\left(t^{\prime \prime}\right), t^{\prime \prime}\right]-Q_{1}\left[s\left(t^{\prime \prime}\right), t^{\prime \prime}\right]\right\}-\rho l\left[\dot{s}_{2}\left(t^{\prime \prime}\right)-\dot{s}_{1}\left(t^{\prime \prime}\right)\right]
$$

where dots indicate time differentiation. Clearly

$$
\dot{s}_{2}\left(t^{\prime \prime}\right) \leq \dot{s}_{1}\left(t^{\prime \prime}\right)
$$

and thus the right-hand side of (17) is positive if at least one of the inequalities in (13) and (18) hold at $t^{\prime \prime}$. But (16) requires, together with $T_{2}=T_{1}=T_{m}$ at $x=s\left(t^{\prime \prime}\right), t=t^{\prime \prime}$, that the left-hand side of (17) be negative; hence a contradiction arises under the present assumption that $s_{1}=s_{2}$ at $t^{\prime \prime}$. The special case of

$$
Q_{2}=Q_{1}, \quad \dot{s}_{2}=\dot{s}_{1} \quad \text { at } \quad x=s\left(t^{\prime \prime}\right), \quad t=t^{\prime \prime}
$$

must now be considered; however for this case the proof is identical with that of [8], with the term $d Q / d t$ being replaced by the total derivative along $x=s(t)$, namely

$$
d Q / d t=\partial Q / \partial t+\dot{s} \partial Q / \partial x
$$

and, by noting that if either condition (a) or (b) of the uniqueness theorem holds, then

$$
d Q_{2}\left[s\left(t^{\prime \prime}\right), t^{\prime \prime}\right] / d t \geq d Q_{1}\left[s\left(t^{\prime \prime}\right), t^{\prime \prime}\right] / d t .
$$

In all cases then, a contradiction occurs if (12b) is assumed to fail, and thus the theorem is proved. The validity of (12a) then follows immediately from the minimum theorem [8], [11].

5. Examples. The conditions for uniqueness previously derived are of course merely sufficient, and the examples given below indeed indicate that they are not necessary. 
The solution of the melting problem formulated in Sec. 2 was derived in [10], for very short times after the start of melting, by means of the "embedding technique". In this technique the temperature in the region $s(t)<x<L$ is taken as that occurring within $0<x<L$ under suitable heating conditions at $x=0$. It is important for the subsequent discussion to note that, since the temperature in $0<x<L$ was expressed as an integral on the Green's function for that region, negative values of $s$ are neither physically admissible, nor do they correspond to temperatures with the required degree of continuity. In discussing uniqueness, therefore, we need only consider positive real functions $s(t)$.

With the dimensionless notation of [10], namely

$$
\begin{aligned}
y & =1-t / t_{m} ; \quad \xi(y)=s(t) / 2 \sqrt{\kappa t_{m}} ; \quad m=\sqrt{\pi} c T_{m} / 2 l_{i} \\
a_{11} & =\left[\partial\left(T / T_{m}\right) / \partial y\right]_{x=0 ; y=0-} ; \quad Q_{0}=\sqrt{\pi} k T_{m} / 2 \sqrt{\kappa t_{m}}
\end{aligned}
$$

where $\kappa$ is the thermal diffusivity, the starting solution is found to be

$$
\xi(y)=\int_{0}^{y} \frac{\Delta Q\left(y^{\prime}\right)}{Q_{0}} d y^{\prime}+\frac{4 m}{3 \pi} a_{11} y^{3 / 2}, \quad y \ll 1
$$

provided that $a_{11} \neq 0$, that the temperature at $x=0$ is continuous at $t=t_{m}$, and that

$$
\begin{gathered}
\lim _{y \rightarrow 0}\left[y^{1 / 2} \Delta Q(y)\right] \neq C, \quad \text { a positive constant; } \\
\Delta Q(y) \equiv Q[\xi(y), y]-Q[0,0-] .
\end{gathered}
$$

Eq. (22) gives only the highest order-of-magnitude term in the solution; hence if the two terms on the right-hand side are of different orders, only the larger one must be retained. It follows that

$$
\xi=4 m a_{11} y^{3 / 2} / 3 \pi \quad \text { if } \quad \Delta Q<C y^{1 / 2}, \quad y \ll 1,
$$

so that in this case the starting solution is identical with that valid for heat inputs independent of $x$.

Consider now the class of problems in which the two terms on the right-hand side of (22) are of the same order, i.e.

$$
\xi(y)=c y^{3 / 2} ; \quad \frac{\Delta Q(y)}{Q_{0}}=\sum_{n} a_{n} \xi^{n} y^{(1-3 n) / 2}=\sum_{n} a_{n} c^{n} y^{1 / 2}
$$

where any combination of values of $n$ may be used in the summation. Note that condition (a) of the uniqueness theorem is met only if $n=0$, condition (b) if all $a_{n}$ 's are nonpositive, and condition (c) is never met.

Substitution into Eq. (22) gives an equation for $c$ as

$$
\frac{3}{2} c=\sum_{n} a_{n} c^{n}+\frac{2 m a_{11}}{\pi} .
$$

If only one term is kept and $n=0$, the uniqueness theorem predicts that only one real positive root for $c$ exists, and this is indeed the case. If $n=\frac{1}{2}$, Eq. (25) is quadratic in $\sqrt{ } \bar{c}$, and

$$
3 \sqrt{c}=a_{1 / 2} \pm \sqrt{a_{1 / 2}^{2}+12 m a_{11} / \pi} .
$$


Thus only one real positive root is found, in conformity with the uniqueness theorem if $a_{1 / 2} \leq 0$; the theorem gives no information if $a^{1 / 2}>0$. Similar conclusions are reached with $n=1$.

If two terms in the summation are used, with $n=\frac{1}{3}$ and $n=\frac{2}{3}$, then $c^{1 / 3}$ is the solution of the cubic

$$
\frac{3}{2} c-a_{2 / 3} c^{2 / 3}-a_{1 / 3} c^{1 / 3}-\left(2 m a_{11} / \pi\right)=0,
$$

which has, from Descartes' rule of signs, at most one positive real root if $a_{2 / 3}$ and $a_{1 / 3}$ are of like sign and $a_{1 / 3}>0$, but will have three positive real roots for suitably chosen $a_{2 / 3}>0$ and $a_{1 / 3}<0$.

More interesting results are obtained with $n=2$, in which case $\Delta Q / Q_{0}=a_{2} \xi^{2} y^{-5 / 2}$ and

$$
c=\frac{3}{4 a_{2}}\left[1 \pm \sqrt{1-\frac{32 m a_{2} a_{11}}{9 \pi}}\right] .
$$

Three cases may be distinguished (cf. Fig. 1):

(1) $a_{2} \leq 0$ : One positive real root, in agreement with condition (b) of the uniqueness theorem;

(2) $0<a_{2}<9 \pi /\left(32 m a_{11}\right)$ : Two positive real roots; the solution is not unique; no contradiction with the uniqueness theorem.

(3) $a_{2}>a \pi /\left(32 m a_{11}\right)$ : Two complex roots; no real solution to the problem under the conditions stated. On the other hand, if condition (22a) is not excluded, then the solution is [10] $\xi \propto y^{1 / 2}$; but

$$
\frac{\Delta Q(y)}{Q_{0}}=C y^{-1 / 2}=a_{2} \xi^{2} y^{-5 / 2} \quad \text { or } \quad \xi \propto y
$$

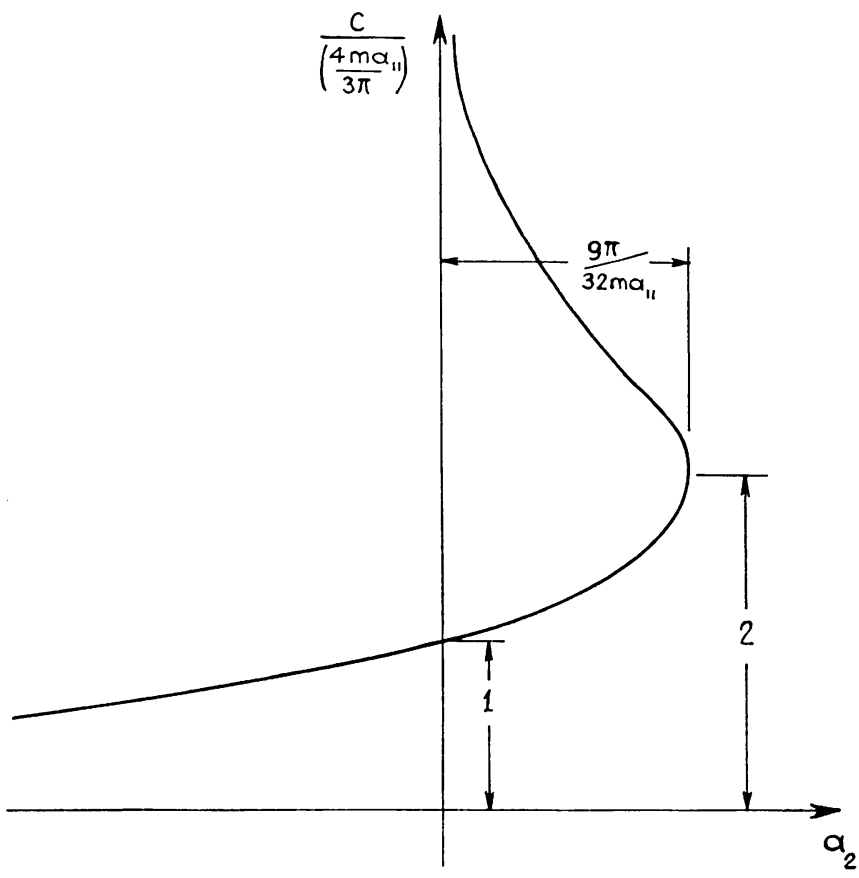

FIG. 1. Real positive roots of Eq. (19). 
so that again no solution is possible. This is a phenomenon not heretofore observed, namely that, with certain types of space- and time-dependent heat inputs applied at a sufficiently high rate, melting will not occur at all. Solutions will of course exist if the solid becomes superheated, and perhaps if the physically unrealistic requirement of instantaneous liquid removal is replaced by ablation at a finite rate; certainly, in fact, no difficulty arises if the liquid remains stationary, in which case the $x$-dependence of the applied heat input is irrelevant since the heated surface does not move. A full examination of these questions would however require a study of the existence of solution in a different physical problem than the one presently being considered.

\section{REFERENCES}

[1] B. A. Boley, A method of heat-conduction analysis of melting and solidifying slabs, J. Math. Phys. 40, 300-313 (1961)

[2] - Upper and lower bounds in problems of melting or solidifying slabs, Quart. J. Mech. Appl. Math. 17, 253-269 (1964)

[3] T. S. Wu and B. A. Boley, Bounds in melting problems with arbitrary rates of liquid removal, SIAM J. Appl. Math. 14, 306-323 (1966)

[4] S. J. Citron, Heat conduction in melting slab, J. Aero. Sci. 27, 219-228 (1960)

[5] G. W. Sutton, The hydrodynamics and heat conduction of a melting surface, J. Aero. Sci. 25, 29-32, 36 (1958)

[6] N. J. Hoff, Structures and materials for finite lifetime, Advances Aero. Sci., 2, Pergamon Press, 1958

[7] B. A. Boley, The analysis of problems of heat conduction and melting, in High temperature structures and materials, Proc. Symp. on Naval Structural Mechanics, Pergamon Press, New York, pp. 260315,1964

[8] — Upper and lower bounds for the solution of a melting problem, Quart. Appl. Math. 21, 1-11 (1963)

[9] - Estimate of errors in approximate temperature and thermal stress calculations, Proc. XI Int. Congr. Appl. Mech., Munich, 586-596, Springer-Verlag, Berlin, 1964

[10] — A general starting solution for melting and solidifying slabs, Int. J. Eng. Sci. 6, 89-111 (1968)

[11] M. Picone, Sul problema della progagazione del calore in un mezzo privo di frontiera, conduttore, isotropo e omogeneo, Math. Ann. 101, 701 (1929) 